\title{
Stabilizing Transmission Intervals and Delays for Linear Time-Varying Control Systems: The Large Delay Case
}

\author{
Domagoj Tolić and Sandra Hirche
}

\begin{abstract}
The goal of this paper is to compute transmission intervals and delays that provably stabilize Linear TimeVarying (LTV) control systems in the presence of disturbances. In other words, given some signal delay existent in a control system, we determine rates at which information between the controller and plant need to be exchanged such that the closedloop system is stable (in some appropriate sense). Depending on information noise and disturbances, the computed transmission intervals lead to stability, asymptotic stability and $\mathcal{L}_{p^{-}}$ stability with bias. The proposed notion of $\mathcal{L}_{p}$-stability with bias integrates noisy information into our stability analysis. The salient feature of our emulation-based methodology is the consideration of delays that are greater than transmission intervals. This feature stems from impulsive delayed system modeling and Lyapunov-Razumikhin techniques employed in the paper. Our methodology is demonstrated on the benchmark problem of batch reactor and compared with a related work.
\end{abstract}

\section{INTRODUCTION}

Nowadays control systems are increasingly implemented in digital technology due to its notable advantages in terms of performance, flexibility and affordability over analog technology [1]. At the same time, digital technology entails delayed and sampled signals between plants and controllers in control loops. Furthermore, sensors and actuators are becoming more spatially distributed which additionally augments delays [2]. Delayed and sampled information can have detrimental effects on the control system performance and might even lead to instability [3]-[12]. Accordingly, control laws designed on the premise of continuous and instantaneous information exchange ought to be thoughtfully transferred into a digital and networked control setting.

The present paper takes up the emulation-based approach presented in [4] and [7] when investigating effects of delayed and intermittent data. In emulation-based approaches one first designs a controller without taking into account the communication network. Subsequently, one determines how often control and sensor signals have to be transmitted/exchanged

D. Tolic is with the University of Zagreb, Faculty of Electrical Engineering and Computing, Unska 3, 10000 Zagreb, Croatia. S. Hirche is with the Institute for Information-oriented Control, Technische Universität München, Arcisstraße 21, D-80290 München, Germany. domagoj.tolicefer.hr, hirchedtum.de

This work has been supported by the European Community's Seventh Framework Programme under grant No. 285939 (ACROSS). D. Tolić is partially supported by the Air Force Research Laboratory under agreemen number FA8655-13-1-3055. The U.S. Government is authorized to reproduce and distribute reprints for Governmental purposes notwithstanding any copyright notation thereon.

The views and conclusions contained herein are those of the authors and should not be interpreted as necessarily representing the official policies or endorsements, either expressed or implied, of the Air Force Research Laboratory or the U.S. Government. over the network so that the closed-loop system remains stable (in some appropriate sense such as asymptotically stable or $\mathcal{L}_{p}$-stable). In other words, even though our modeling can incorporate various delay compensation or model-based control schemes (consult [6], [8], [11], [13] for more) that produce greater stabilizing delays and transmission intervals, we do not explicitly design such schemes but rather aim our attention at analyzing the robustness of a given control system to realistic networking artifacts.

When analyzing sampled-data systems with delays, it is often required that delays are smaller than the transmission/sampling intervals (see [7], [11], [14] and the references therein). This case is known as the small delay case. Such a requirement might be overly restrictive for many control systems. In order to circumvent this prohibitive requirement, our paper employs impulsive delayed system modeling with Lyapunov-Razumikhin techniques and proposes a framework for computation of Maximally Allowable Transmission Intervals (MATIs) that stabilize control systems even for large delays. Furthermore, we propose the notion of $\mathcal{L}_{p}$-stability with bias as a way to integrate noisy information into stability results. As far as Linear Time-Varying (LTV) dynamics and one packet transmissions are concerned, the present paper is an improvement upon the state-of-the-art approach of [7] with respect to large delays and noisy data. According to [9], one packet transmissions correspond to the setting in which all the information are sent together in a single packet. Notice that MATIs reduce requirements posed on sensors and processors in control systems without compromising stability or the performance of control systems. As in [7], we quantify the control system performance by means of $\mathcal{L}_{p}$-gains.

The main contributions of this paper are fourfold: a) the design of MATIs for the large delay case; b) the LyapunovRazumikhin-based procedure for rendering $\mathcal{L}_{p}$-stability of LTV impulsive delayed systems and computing the associated $\mathcal{L}_{p}$-gains; c) the consideration of distorted information; and d) the novel result relating asymptotic stability and $\mathcal{L}_{p^{-}}$ stability for a class of LTV impulsive delayed systems.

The reminder of the paper is organized as follows. Section II presents notation and various stability notions for impulsive delayed systems. Depending on the assumptions imposed on LTV control systems, Section III states the problem of finding transmission intervals and delays that stabilize such systems in some appropriate sense. Our methodology to solve the problem of interest is found in Section IV. This methodology is illustrated and verified in Section V using the well-studied batch reactor example. Conclusions and future challenges are in Section VI. 


\section{PRELIMINARIES}

\section{A. Notation}

To simplify the notation, we use $(x, y):=\left[\begin{array}{ll}x^{\top} & y^{\top}\end{array}\right]^{\top}$. The dimension of a vector $x$ is denoted $n_{x}$. Next, let $f$ : $\mathbb{R} \rightarrow \mathbb{R}^{n}$ be a Lebesgue measurable function on $[a, b] \subset \mathbb{R}$. We use

$$
\|f[a, b]\|_{p}:=\left(\int_{[a, b]}\|f(s)\|^{p} \mathrm{~d} s\right)^{\frac{1}{p}}
$$

to denote the $\mathcal{L}_{p}$ norm of $f$ when restricted to the interval $[a, b]$. If the corresponding norm is finite, we write $f \in$ $\mathcal{L}_{p}[a, b]$. In the above expression, $\|\cdot\|$ refers to the Euclidean norm of a vector. If the argument of $\|\cdot\|$ is a matrix $A$, then it denotes the induced 2-norm of $A$. The $n$-dimensional vector with all zero entries is denoted $\mathbf{0}_{n}$. Likewise, the $n$ by $m$ matrix with all zero entries is denoted $\mathbf{0}_{n \times m}$. In addition, $\mathbb{R}_{+}^{n}$ denotes the nonnegative orthant. The natural numbers are denoted $\mathbb{N}$ or $\mathbb{N}_{0}$ when zero is included.

Left-hand and right-hand limits are denoted $x\left(t^{-}\right)=$ $\lim _{t^{\prime} \nearrow_{t}} x\left(t^{\prime}\right)$ and $x\left(t^{+}\right)=\lim _{t^{\prime} \searrow t} x\left(t^{\prime}\right)$, respectively. Next, for a set $\mathcal{S} \subseteq \mathbb{R}^{n}$, let $\operatorname{PC}([a, b], \mathcal{S})=\{\phi:[a, b] \rightarrow$ $\mathcal{S} \mid \phi(t)=\phi\left(t^{+}\right)$for every $t \in[a, b), \phi\left(t^{-}\right)$exists in $\mathcal{S}$ for all $t \in(a, b]$ and $\phi\left(t^{-}\right)=\phi(t)$ for all but at most a finite number of points $t \in(a, b]\}$. For $\psi \in P C([a-d, a], \mathcal{S})$, where $d \geq 0$, the norm of $\psi$ is defined by $\|\psi\|_{d}=$ $\sup _{a-d \leq s \leq a}\|\psi(s)\|$.

\section{B. Impulsive Delayed Systems}

In this paper, we consider impulsive delayed systems

$$
\Sigma\left\{\begin{aligned}
\chi\left(t^{+}\right) & =h_{\chi}(t, \chi(t), \chi(t-d)) \\
\dot{\chi}(t) & =f_{\chi}(t, \chi(t), \chi(t-d), \omega) \\
y & =\ell_{\chi}(t, \chi, \omega)
\end{aligned}\right\} \quad \begin{aligned}
& t \in \mathcal{T} \\
& \text { otherwise },
\end{aligned}
$$

where $\chi$ is the state, $\omega$ is the input, $y$ is the output and $d \geq 0$ is the time delay. The functions $f_{\chi}$ and $h_{\chi}$ are regular enough to guarantee forward completeness of solutions which, given initial condition $\psi_{\chi} \in P C\left(\left[t_{0}-d, t_{0}\right], \mathbb{R}^{n_{\chi}}\right)$ and initial time $t_{0}$, are given by right-continuous functions $t \mapsto \chi(t) \in$ $P C\left(\left[t_{0}-d, \infty\right], \mathbb{R}^{n_{\chi}}\right)$. Jumps of the state are denoted $\chi\left(t^{+}\right)$ and occur at each $t \in \mathcal{T}:=\left\{t_{1}, t_{2}, \ldots\right\}$, where $t_{i}<t_{i+1}$, $i \in \mathbb{N}_{0}$. The value of the state after a jump is given by $\chi\left(t^{+}\right)$ for each $t \in \mathcal{T}$. For a comprehensive discussion regarding the solutions to (1) considered herein, refer to [15, Chapter 2 $\& 3$ ]. Even though the considered solutions to (1) allow for jumps at $t_{0}$, we exclude such jumps in favor of notational convenience.

Definition 1 (Uniform Global Stability): For $\omega \equiv \mathbf{0}_{n_{\omega}}$, the system $\Sigma$ is said to be Uniformly Globally Stable (UGS) if for any $\epsilon>0$ there exists $\delta(\epsilon)>0$ such that, for each $t_{0} \epsilon$ $\mathbb{R}$ and each $\psi_{\chi} \in P C\left(\left[t_{0}-d, t_{0}\right], \mathbb{R}^{n_{\chi}}\right)$ satisfying $\left\|\psi_{\chi}\right\|_{d}<$ $\delta(\epsilon)$, each solution $t \mapsto \chi(t) \in P C\left(\left[t_{0}-d, \infty\right], \mathbb{R}^{n_{\chi}}\right)$ to $\Sigma$ satisfies $\|\chi(t)\|<\epsilon$ for all $t \geq t_{0}$ and $\delta(\epsilon)$ can be chosen such that $\lim _{\epsilon \rightarrow \infty} \delta(\epsilon)=\infty$.

Definition 2 (Uniform Global Asymptotic Stability): For $\omega \equiv \mathbf{0}_{n_{\omega}}$, the system $\Sigma$ is said to be Uniformly Globally
Asymptotically Stable (UGAS) if it is UGS and uniformly globally attractive, i.e., for each $\eta, c>0$ there exists $T(\eta, c)>0$ such that $\|\chi(t)\|<\eta$ for every $t \geq t_{0}+T(\eta, c)$ and every $\left\|\chi\left(t_{0}\right)\right\|<c$.

Definition 3 (Uniform Global Exponential Stability):

For $\omega \equiv \mathbf{0}_{n_{\omega}}$, the system $\Sigma$ is said to be Uniformly Globally Exponentially Stable (UGES) if there exist positive constants $\lambda$ and $M$ such that, for each $t_{0} \in \mathbb{R}$ and each $\psi_{\chi} \in P C\left(\left[t_{0}-d, t_{0}\right], \mathbb{R}^{n_{\chi}}\right)$, each solution $t \mapsto \chi(t) \in P C\left(\left[t_{0}-d, \infty\right], \mathbb{R}^{n_{\chi}}\right)$ to $\Sigma$ satisfies $\|\chi(t)\| \leq M\left\|\psi_{\chi}\right\|_{d} e^{-\lambda\left(t-t_{0}\right)}$ for each $t \geq t_{0}$.

Definition $4\left(\mathcal{L}_{p}\right.$-Stability with Bias $\left.b\right)$ : Let $p \in[1, \infty]$. The system $\Sigma$ is $\mathcal{L}_{p}$-stable with bias $b(t) \equiv b \geq 0$ from $\omega$ to $y$ with gain $\gamma \geq 0$ if there exists $K \geq 0$ such that, for each $t_{0} \in \mathbb{R}$ and each $\psi_{\chi} \in P C\left(\left[t_{0}-d, t_{0}\right], \mathbb{R}^{n_{\chi}}\right)$, each solution to $\Sigma$ from $\psi_{\chi}$ at $t=t_{0}$ satisfies $\left\|y\left[t_{0}, t\right]\right\|_{p} \leq$ $K\left\|\psi_{\chi}\right\|_{d}+\gamma\left\|\omega\left[t_{0}, t\right]\right\|_{p}+\left\|b\left[t_{0}, t\right]\right\|_{p}$ for each $t \geq t_{0}$.

Definition 5 ( $\mathcal{L}_{p}$-Detectability): Let $p \in[1, \infty]$. The state $\chi$ of $\Sigma$ is $\mathcal{L}_{p}$-detectable from $(y, \omega)$ with gain $\gamma \geq 0$ if there exists $K \geq 0$ such that, for each $t_{0} \in \mathbb{R}$ and each $\psi_{\chi} \in$ $P C\left(\left[t_{0}-d, t_{0}\right], \mathbb{R}^{n_{\chi}}\right)$, each solution to $\Sigma$ from $\psi_{\chi}$ at $t=t_{0}$ satisfies $\left\|\chi\left[t_{0}, t\right]\right\|_{p} \leq K\left\|\psi_{\chi}\right\|_{d}+\gamma\left\|y\left[t_{0}, t\right]\right\|_{p}+\gamma\left\|\omega\left[t_{0}, t\right]\right\|_{p}$ for each $t \geq t_{0}$.

Definitions 1, 2 and 3 are motivated by [16], while Definition 5 is inspired by [4]. Definition 4 is motivated by [4] and [17]. When $b=0$, we say " $\mathcal{L}_{p}$-stability" instead of " $\mathcal{L}_{p}$-stability with bias 0".

\section{Problem Statement}

Consider a feedback control system consisting of an LTV plant

$$
\begin{aligned}
\dot{x}_{p} & =A_{p}(t) x_{p}+B_{p}(t) u+\omega_{p}, \\
y & =C_{p}(t) x_{p},
\end{aligned}
$$

and an LTV controller

$$
\begin{aligned}
\dot{x}_{c} & =A_{c}(t) x_{c}+B_{c}(t) y+\omega_{c}, \\
u & =C_{c}(t) x_{c},
\end{aligned}
$$

where $x_{p} \in \mathbb{R}^{n_{p}}$ and $x_{c} \in \mathbb{R}^{n_{c}}$ are the states, $y \in \mathbb{R}^{n_{y}}$ and $u \in \mathbb{R}^{n_{u}}$ are the outputs, and $\left(u, \omega_{p}\right) \in \mathbb{R}^{n_{u}} \times \mathbb{R}^{n_{\omega_{p}}}$ and $\left(y, \omega_{c}\right) \in \mathbb{R}^{n_{y}} \times \mathbb{R}^{n_{\omega_{c}}}$ are the inputs of the plant and controller, respectively, where $\omega_{p}$ and $\omega_{c}$ are disturbances to (and/or modeling uncertainties of) the plant and controller, respectively. We assume that all time-varying matrices in (2) - (3) are element-wise bounded right-continuous functions. In addition, we require that the entries of $C_{p}(t)$ and $C_{c}(t)$ are piecewise continuously differentiable with rightcontinuous and bounded first derivatives. Along with the absence of Zeno sampling (which is to be shown later on), these properties of $A_{p}(t), B_{p}(t), C_{p}(t), A_{c}(t), B_{c}(t)$ and $C_{c}(t)$ suffice to ensure forward completeness (as well as uniqueness) of the solutions (consult [15, Chapter 3] and [18] for more).

Next, we model the connections between the plant and the controller as communication networks over which intermittent exchange of information takes place. Figure 1 depicts 


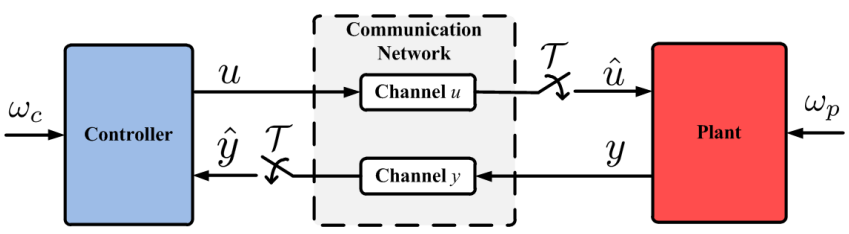

Fig. 1. A diagram of a control system with the plant and controller interacting over a communication network with intermittent information updates. The two switches indicate that the information between the plant and controller are exchanged at discrete time instants belonging to a set $\mathcal{T}$.

this setting, where the value of $u$ computed by the controller that arrives to the plant is denoted $\hat{u}$. Similarly, the values of $y$ that the controller actually receives are denoted $\hat{y}$. In this setting, the quantity $\hat{u}$ is the input fed to the plant (2) while the quantity $\hat{y}$ is the measurement of $y$ received by the controller (3).

To study the properties of the feedback control system in Figure 1, we define

$$
e=\left[\begin{array}{l}
e_{y}(t) \\
e_{u}(t)
\end{array}\right]:=\left[\begin{array}{l}
\hat{y}(t)-y(t-d) \\
\hat{u}(t)-u(t-d)
\end{array}\right],
$$

where $d \geq 0$ represents the network-induced delay ${ }^{1}$. As given by (4), we assume this network-induced delay $d$ is the same when transmitting $u$ or $y$ (primarily in order to simplify the subsequent exposition). This assumption, which is often found in the literature, can be accomplished via the Controller Area Network (CAN) protocol, time-stamping of data and introduction of buffers at receiver ends (refer to [2] and the references therein). The above definition of the error vector allows us to take into account large delays (cf. [7]).

To model intermittent transmission (or sampling) of the values of $y$ and $u$, the quantities $\hat{y}$ and $\hat{u}$ are updated at time instances $t_{1}, t_{2}, \ldots, t_{i}, \ldots \in \mathcal{T}$, i.e., ${ }^{2}$

$$
\left.\begin{array}{l}
\hat{y}\left(t_{i}^{+}\right)=y\left(t_{i}-d\right)+h_{y}\left(t_{i}-d\right) \\
\hat{u}\left(t_{i}^{+}\right)=u\left(t_{i}-d\right)+h_{u}\left(t_{i}-d\right)
\end{array}\right\} \quad t_{i} \in \mathcal{T},
$$

where $h_{y}: \mathbb{R} \rightarrow \mathbb{R}^{n_{y}}$ and $h_{u}: \mathbb{R} \rightarrow \mathbb{R}^{n_{u}}$ are $\mathcal{L}_{\infty^{-}}$ functions and model noise or channel disturbances. The consideration of noisy/distorted data produces non-zero bias $b$ in Definition 4. In order to provide a better comparison with the related works, this paper assumes that the received values of $y$ and $u$ given by $\hat{y}$ and $\hat{u}$, respectively, remain constant in between updates. In other words, for each $t \in\left[t_{0}, \infty\right) \backslash \mathcal{T}$

\footnotetext{
${ }^{1}$ In case of time-invariant static controllers with $\omega_{c} \equiv \mathbf{0}_{n_{\omega_{c}}}$, the effect of nontrivial execution times of the control law can readily be embedded in $d$. In order to account for nontrivial execution times of other types of controllers, slight modifications of the proposed modeling approach are needed.

${ }^{2}$ The formulation of the update law in (5) implies that the jump times at the controller and plant end coincide (the so-called one packet transmission problem [9]). Hence, in general, the framework presented herein does not incorporate scheduling protocols. Exceptions are scenarios in which some subsets of $e$ are decoupled from other subsets of $e$ (e.g., $\dot{e}(t)$ with a block diagonal state matrix). In such scenarios, each subset of $e$ can experience jumps at time instants that are independent of other subsets' jump instants (Section V provides such an example). Scheduling protocols will be addressed in our upcoming publications.
}

we have

$$
\dot{\hat{y}}=0, \quad \dot{\hat{u}}=0
$$

which is known as the Zero-Order Hold ( $\mathrm{ZOH})$ strategy. However, one can immediately employ model-based control ideas in (6) in order to further extend transmission intervals (consult [8] for more). Let us point out that the $\mathrm{ZOH}$ strategy is an integral part of the small-delay approach in [7]; hence, the $\mathrm{ZOH}$ strategy in [7] cannot be trivially relaxed.

The following standing assumption summarizes the properties imposed upon the feedback control system in Figure 1 throughout this paper.

Assumption 1: The jump times at the controller and plant end coincide. The set of sampling instants is given by $\mathcal{T}:=$ $\left\{t_{1}, t_{2}, \ldots, t_{i}, \ldots\right\}$, where $\tau=t_{i+1}-t_{i}$ for each $i \in \mathbb{N}_{0}$. In addition, the network-induced delay when transmitting $u$ is the same as the network-induced delay when transmitting $y$ and is denoted $d$.

As our intuition suggests, a typical closed-loop system (2) (3) might be robust (in the $\mathcal{L}_{p}$-stability sense) only for some values of $d$, i.e., "small enough" values of $d$. We refer to such delays as admissible delays. For a precise definition of admissible delays, see the following section. Given some admissible delay $d$, the maximal $\tau$ which renders stability (in some appropriate sense) of the closed-loop system (2) - (3) is called MATI and is denoted $\bar{\tau}$. We are now ready to state the main problem studied in this paper.

Problem 1: Given an admissible delay $d$, determine the MATI $\bar{\tau}$ to update $(\hat{y}, \hat{u})$ such that the control system (2)(3) is UGS, GAS or $\mathcal{L}_{p}$-stable with bias and a prespecified $\mathcal{L}_{p}$-gain.

As opposed to [7], Problem 1 starts with an admissible delay and then seeks out the corresponding MATI whilst [7] starts with the MATI for the delay-free setting and than seeks out the corresponding Maximally Allowable Delay (MAD).

\section{Methodology}

\section{A. Rewriting the Closed-Loop System}

Inspired by the approach in [4], our solution to Problem 1 first rewrites the closed-loop system (2)-(3) as follows:

$$
\begin{aligned}
& \left.\begin{array}{l}
x\left(t^{+}\right)=x(t) \\
e\left(t^{+}\right)=h(t-d)
\end{array}\right\} \quad t \in \mathcal{T} \\
& \left.\begin{array}{rl}
\dot{x} & =f(t, x, e, \omega, d) \\
\dot{e} & =g(t, x, e, \omega, d)
\end{array}\right\} \quad \text { otherwise, }
\end{aligned}
$$




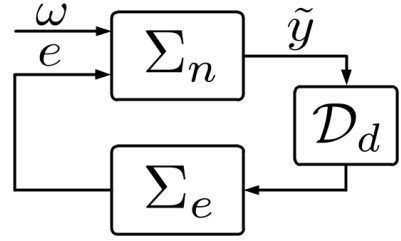

Fig. 2. Interconnection of the nominal system $\Sigma_{n}$ and the output error system $\Sigma_{e}$. The block $\mathcal{D}_{d}$ denotes the time-delay operator.

where $x:=\left(x_{p}, x_{c}\right), \omega:=\left(\omega_{p}, \omega_{c}\right)$, and functions $f, g$ and $h$ are given by

$$
\begin{aligned}
& f(t, x, e, \omega, d):=\left[\begin{array}{l}
f_{1}(t, x, e, \omega, d) \\
f_{2}(t, x, e, \omega, d)
\end{array}\right]= \\
& \quad=\left[\begin{array}{l}
A_{p}(t) x_{p}(t)+B_{p}(t) e_{u}(t)+B_{p}(t) C_{c}(t-d) x_{c}(t-d)+\omega_{p} \\
A_{c}(t) x_{c}(t)+B_{c}(t) e_{y}(t)+B_{c}(t) C_{p}(t-d) x_{p}(t-d)+\omega_{c}
\end{array}\right], \\
& h\left(t_{i}-d\right)=\left[\begin{array}{l}
h_{y}\left(t_{i}-d\right) \\
h_{u}\left(t_{i}-d\right)
\end{array}\right], \\
& g(t, x, e, \omega, d)= \\
& \quad=\left[\begin{array}{l}
-\frac{\partial C_{p}(t-d)}{\partial t} x_{p}(t-d)-C_{p}(t-d) f_{1}(t-d, x, e, \omega, d) \\
-\frac{\partial c_{c}(t-d)}{\partial t} x_{c}(t-d)-C_{c}(t-d) f_{2}(t-d, x, e, \omega, d)
\end{array}\right] .
\end{aligned}
$$

Let us rewrite $(8 \mathrm{c})$ as follows:

$g(t, x, e, \omega, d)=-\left[\begin{array}{ll}\mathbf{0}_{n_{y} \times n_{u}} & C_{p} B_{p}(t-d) \\ C_{c} B_{c}(t-d) & \mathbf{0}_{n_{u} \times n_{y}}\end{array}\right] e(t-d)+\tilde{y}(t-d, x, \omega, d)$,

where

$$
\begin{aligned}
& \tilde{y}(t, x, \omega, d):= \\
& {\left[\begin{array}{c}
-\frac{\partial C_{p}(t)}{\partial t} x_{p}(t)-C_{p}(t)\left(A_{p}(t) x_{p}(t)+B_{p}(t) C_{c}(t-d) x_{c}(t-d)+\omega_{p}(t)\right) \\
-\frac{\partial C_{c}(t)}{\partial t} x_{c}(t)-C_{c}(t)\left(A_{c}(t) x_{c}(t)+B_{c}(t) C_{p}(t-d) x_{p}(t-d)+\omega_{c}(t)\right)
\end{array}\right] .}
\end{aligned}
$$

Now, the delayed dynamics

$$
\begin{array}{lll}
x\left(t^{+}\right)=x(t) & \{t \in \mathcal{T} \\
\dot{x}=f(t, x, e, \omega, d) & \} & \text { otherwise, }
\end{array}
$$

with input $(e, \omega)$ and output $\tilde{y}$ are termed the nominal system $\Sigma_{n}$, and the impulsive delayed dynamics

$$
\begin{aligned}
& \left.e\left(t^{+}\right)=h(t-d) \quad\right\} \quad t \in \mathcal{T} \\
& \dot{e}=g(t, x, e, \omega, d) \quad\} \quad \text { otherwise, }
\end{aligned}
$$

with input $\tilde{y}$ and output $e$ are termed the error system $\Sigma_{e}$. In other words, the systems $\Sigma_{n}$ and $\Sigma_{e}$ are interconnected as shown in Figure 2, where the time-delay operator $\mathcal{D}_{d}$ delays the input $\tilde{y}(t, x, \omega, d)$ for $d$ time units, i.e.,

$$
\mathcal{D}_{d}(\tilde{y}(t, x, \omega, d)):=\tilde{y}(t-d, x, \omega, d) .
$$

We point out that the $\mathcal{L}_{p}$-gain of $\mathcal{D}_{d}$ is unity [5].

Because the nominal system $\Sigma_{n}$ is something that is given in emulation-based approaches, we assume that the controller (3) is designed to yield $\mathcal{L}_{p}$-stability of $\Sigma_{n}$. Let $\gamma_{n}$ denote the corresponding $\mathcal{L}_{p}$-gain. As expected, a different $d$ typically results in a different $\gamma_{n}$. In general, controllers yield $\mathcal{L}_{p^{-}}$ stability of $\Sigma_{n}$ (i.e., $\gamma_{n}$ is finite) only for some delays $d$. This observation gives rise to the following definition:
Definition 6 (Admissible Delays): Delays $d$ for which the system $\Sigma_{n}$ is $\mathcal{L}_{p}$-stable from $(e, \omega)$ to $\tilde{y}$ are admissible delays. The maximal such delay is labeled $\bar{d}$.

On the other hand, $\mathcal{L}_{p}$-stability with bias of the error system $\Sigma_{e}$ depends on $\mathcal{T}$. In what follows, we design the set $\mathcal{T}$ such that $\Sigma_{e}$ is $\mathcal{L}_{p}$-stable with bias and that the associated $\mathcal{L}_{p}$-gain, denoted $\gamma_{e}$, satisfies $\gamma_{n} \gamma_{e}<1$. Afterwards, we invoke the small-gain theorem [16, Chapter 5] to infer $\mathcal{L}_{p}$-stability with bias from $\omega$ to $(\tilde{y}, e)$. Provided that $x$ is $\mathcal{L}_{p}$-detectable from $(\tilde{y}, e, \omega)$, one obtains $\mathcal{L}_{p}$-stability with bias from $\omega$ to $(x, e)$. Hence, the closed-loop system (2)-(3) is $\mathcal{L}_{p}$-stable with bias.

\section{B. $\mathcal{L}_{p}$-Stability with Bias of Impulsive Delayed LTV Systems}

Note that $\Sigma_{e}$, given by (12), is an impulsive delayed LTV system. To the best of our knowledge, $\mathcal{L}_{p}$-stability of such systems is still an open problem. To that end, let us first establish UGES of

$$
\left.\begin{array}{rl}
e\left(t^{+}\right) & =\mathbf{0}_{n_{e}}
\end{array}\right\} t \in \mathcal{T} \quad(13
$$

which is the homogeneous system associated with $\Sigma_{e}$. In order to state the following theorem, we define

$$
l:=\left(\sup _{t \in \mathbb{R}}\left\|-\left[\begin{array}{ll}
\mathbf{0}_{n_{y} \times n_{u}} & C_{p} B_{p}(t-d) \\
C_{c} B_{c}(t-d) & \mathbf{0}_{n_{u} \times n_{y}}
\end{array}\right]\right\|\right)^{2} .
$$

Theorem 1: Consider a symmetric and positive definite matrix $P \in \mathbb{R}^{n_{e} \times n_{e}}$ with $\lambda_{1}$ and $\lambda_{2}$ being its smallest and largest eigenvalues, respectively. Let $\lambda_{3}$ be the largest eigenvalue of $l P^{-1}$. If there exist constants $\lambda>0, M>1$ and $\lambda_{4} \in(0,1)$ such that the conditions

(i) $\tau\left(\lambda+\lambda_{2}+\lambda_{3} M e^{-\lambda \tau}\right)<\ln M$, and

(ii) $\tau\left(\lambda+\lambda_{2}+\frac{\lambda_{3}}{\lambda_{4}} e^{\lambda d}\right)<-\ln \lambda_{4}$,

hold, then the system (13) is UGES and $\|e(t)\| \leq$ $\sqrt{\frac{\lambda_{2}}{\lambda_{1}} M}\left\|\psi_{e}\right\|_{d} e^{-\frac{\lambda}{2}\left(t-t_{0}\right)}$ for all $t \geq t_{0}$.

The previous result combined with the work presented in [18] results in the following theorem.

Theorem 2: Suppose that (13) is UGES with constants $\lambda>0$ and $M>1$. Then, the system $\Sigma_{e}$, given by (12), is $\mathcal{L}_{p}$-stable with bias from $\tilde{y}$ to $e$ with gain $\gamma_{e}=\frac{2}{\lambda} \sqrt{\frac{\lambda_{2}}{\lambda_{1}} M}$ for each $p \in[1, \infty]$.

Remark 1 (No Zeno): The left-hand sides of conditions (i) and (ii) from Theorem 1 are nonnegative continuous functions of $\tau \geq 0$ that approach $\infty$ as $\tau \rightarrow \infty$. Also, these left-hand sides equal zero when $\tau=0$. Note that both sides of (i) and (ii) are continuous in $\lambda, M, \lambda_{2}, \lambda_{3}, \lambda_{4}$ and $d$. Hence, for every $\lambda, \lambda_{1}, \lambda_{2}>0, \lambda_{3} \geq 0, M>1$, $\lambda_{4} \in(0,1)$ and $d \geq 0$ there exists $\tau>0$ such that (i) and (ii) are satisfied. Finally, since $\gamma_{e}$ is a continuous function of $\lambda_{1}, \lambda_{2}, \lambda$ and $M$, we infer that for every finite $\gamma_{n}>0$ there exists $\tau>0$ such that $\gamma_{n} \gamma_{e} \leq \kappa, \kappa \in(0,1)$. In other words, the unwanted Zeno behavior is avoided and the proposed methodology does not yield continuous feedback that might be impossible to implement. Notice that the $\tau$ yielding $\gamma_{n} \gamma_{e}=\kappa$ is a candidate for $\bar{\tau}$. Depending on $P$, 
$\lambda_{4}, \lambda$ and $M$, the maximal such $\tau$ is in fact $\bar{\tau}$. Lastly, we point out that intertransmission intervals $t_{i+1}-t_{i}, i \in \mathbb{N}_{0}$, do not have to be equal (i.e., periodic transmissions), but merely upper bounded by $\bar{\tau}$.

\section{UGS and GAS from $\mathcal{L}_{p}$-Stability}

Proposition 1: Assume that the interconnection of systems $\Sigma_{n}$ and $\Sigma_{e}$, given by (11) and (12), is $\mathcal{L}_{p}$-stable from $\omega$ to $(x, e)$ for some $p \in[1, \infty)$. Then, this interconnection is GAS.

At the moment, we are trying to verify whether UGAS holds as well. For the case $p=\infty$, UGS of the interconnection $\Sigma_{n}$ and $\Sigma_{e}$ is immediately obtained using the definition of $\mathcal{L}_{\infty}$-norm.

\section{BAtch Reactor CASE StUdy}

According to [7], the batch reactor case study has become a benchmark example in Networked Control Systems (NCS) over the years. Hence, we apply our work to this example and compare it with the approach presented in [7].

Consider the linearized model of an unstable batch reactor given by

$$
\dot{x}_{p}=A_{p} x_{p}+B_{p} u, \quad y=C_{p} x_{p}
$$

where

$$
\begin{aligned}
A_{p} & =\left[\begin{array}{cccc}
1.38 & -0.2077 & 6.715 & -5.676 \\
-0.5814 & -4.29 & 0 & 0.675 \\
1.067 & 4.273 & -6.654 & 5.893 \\
0.048 & 4.273 & 1.343 & -2.104
\end{array}\right], B_{p}=\left[\begin{array}{cc}
0 & 0 \\
5.679 & 0 \\
1.136 & -3.146 \\
1.136 & 0
\end{array}\right], \\
C_{p} & =\left[\begin{array}{llll}
1 & 0 & 1 & -1 \\
0 & 1 & 0 & 0
\end{array}\right],
\end{aligned}
$$

and a PI controller given by

$$
\dot{x}_{c}=A_{c} x_{c}+B_{c} y, \quad u=C_{c} x_{c}+D_{c} y,
$$

where

$$
A_{c}=\left[\begin{array}{ll}
0 & 0 \\
0 & 0
\end{array}\right], B_{c}=\left[\begin{array}{ll}
0 & 1 \\
1 & 0
\end{array}\right], C_{c}=\left[\begin{array}{cc}
-2 & 0 \\
0 & 8
\end{array}\right], D_{c}=\left[\begin{array}{cc}
0 & -2 \\
5 & 0
\end{array}\right] .
$$

In addition, assume that only the plant outputs $y$ are transmitted via the network; hence, $e=e_{y}$ and $e_{u} \equiv \mathbf{0}_{n_{u}}$. Notice that the above controller, unlike (3), includes the matrix $D_{c}$. Nevertheless, the same approach from Section IV-A still applies. Following the development of Section IVA, we obtain

$$
\begin{aligned}
\dot{x}(t)= & {\left[\begin{array}{l}
\dot{x}_{p}(t) \\
\dot{x}_{c}(t)
\end{array}\right]=\left[\begin{array}{cc}
A_{p} & B_{p} C_{c} \\
\mathbf{0}_{2 \times 4} & A_{c}
\end{array}\right] x(t)+} \\
& +\left[\begin{array}{cc}
B_{p} D_{c} C_{p} & \mathbf{0}_{4 \times 2} \\
B_{c} C_{p} & \mathbf{0}_{2 \times 2}
\end{array}\right] x(t-d)+\left[\begin{array}{c}
B_{p} D_{c} \\
B_{c}
\end{array}\right] e(t)+ \\
& +\left[\begin{array}{cccccc}
10 & 0 & 10 & 0 & 0 & 0 \\
0 & 5 & 0 & 5 & 0 & 0
\end{array}\right]^{\top} \omega(t),
\end{aligned}
$$

where the last term including $\omega(t)$ is subsequently added by the authors in [7], and

$$
\begin{aligned}
& \dot{e}(t)=\left[-C_{p} B_{p} D_{c}\right] e(t-d)+ \\
& +\left[\begin{array}{ll}
-C_{p} A_{p} & -C_{p} B_{p} C_{c}
\end{array}\right] x(t-d)+ \\
& +\left[\begin{array}{ll}
-C_{p} B_{p} D_{c} C_{p} & \mathbf{0}_{2 \times 2}
\end{array}\right] x(t-2 d) .
\end{aligned}
$$

We point out that

$$
-C_{p} B_{p} D_{c}=\left[\begin{array}{cc}
15.73 & 0 \\
0 & 11.3580
\end{array}\right]
$$

is a diagonal matrix, and this fact is exploited below. From the previous expressions, we infer that

$$
\begin{aligned}
\tilde{y}(t) & =\left[\begin{array}{ll}
-C_{p} A_{p} & -C_{p} B_{p} C_{c}
\end{array}\right] x(t)+ \\
& +\left[\begin{array}{ll}
-C_{p} B_{p} D_{c} C_{p} & \mathbf{0}_{2 \times 2}
\end{array}\right] x(t-d) .
\end{aligned}
$$

Since the example in [7] does not consider noisy information, one can set $h(t) \equiv \mathbf{0}_{n_{e}}$. In other words, bias $b(t) \equiv b=0$ and all $\mathcal{L}_{2}$-stability results are without bias.

In order to compute $\gamma_{n}$ for $p=2$, we use the software HINFN [19]. However, HINFN is not able to handle delayed states in the output (see (14)). In order to accommodate (14) to HINFN, we first compute an estimate of the $\mathcal{L}_{2}$-gain, say $L_{1}$, from $(e, \omega)$ to $y_{1}:=\left[\begin{array}{ll}-C_{p} A_{p} & -C_{p} B_{p} C_{c}\end{array}\right] x(t)$, and than an estimate of the $\mathcal{L}_{2}$-gain, say $L_{2}$, from $(e, \omega)$ to $y_{2}:=\left[\begin{array}{ll}-C_{p} B_{p} D_{c} C_{p} & \mathbf{0}_{2 \times 2}\end{array}\right] x(t)$. Afterwards, we simply add together those two gains because

$$
\begin{aligned}
\| y_{1}(t)\left[t_{0}, \infty\right)+ & y_{2}(t-d)\left[t_{0}, \infty\right) \|_{p}= \\
& =\left\|y_{1}(t)\left[t_{0}, \infty\right)+y_{2}(t)\left[t_{0}+d, \infty\right)\right\|_{p} \\
\leq & \left\|y_{1}(t)\left[t_{0}, \infty\right)\right\|_{p}+\left\|y_{2}(t)\left[t_{0}, \infty\right)\right\|_{p} \\
\leq & \left(L_{1}+L_{2}\right)\left\|(e, \omega)\left[t_{0}, \infty\right)\right\|_{p},
\end{aligned}
$$

where we used the fact that the $\mathcal{L}_{2}$-gain of the time-delay operator $\mathcal{D}_{d}$ is less than unity and that $y_{2}(t-d)=0$ when $t-d<t_{0}$. According to HINFN, the maximally admissible delay $\bar{d}$ is $40 \mathrm{~ms}$. In addition, we choose the controlled output $z$ given by $z(t)=C x(t)$ where

$$
C=\left[\begin{array}{cccccc}
1 & 0 & 1 & -1 & 0 & 0 \\
0 & 1 & 0 & 0 & 0 & 0
\end{array}\right]
$$

One goal of [7] is to determine pairs $(d, \bar{\tau})$ such that the $\mathcal{L}_{2}$-gain, denoted $\gamma_{z}$, from $\omega$ to $z$ is below a certain value.

When one is interested merely in asymptotic stability, then the input to $\Sigma_{n}$ is $e$ instead of $(e, \omega)$ because, for this particular example, the corresponding $\gamma_{n}$ is smaller (i.e., $\bar{\tau}$ is greater). For UGAS, HINFN suggests that $\bar{d}$ is $2.34 \mathrm{~s}$. However, the corresponding $\bar{\tau}$ is about $10^{-200} \mathrm{~s}$ (which has no practical merit but rather confirms Remark 1). Notice that, since the plant and controller in the batch reactor example are Linear Time-Invariant (LTI) systems, GAS of Proposition 1 is in fact UGAS.

In Tables I and II, a comparison between the methodology presented herein and in [7] is provided. We consider the case without $\omega$, which leads to asymptotic stability, and the case with $\omega$, which leads to $\mathcal{L}_{2}$-stability from $\omega$ to $z$ with a prespecified gain $\gamma_{z}$. In addition, since $e_{1}$ and $e_{2}$ are decoupled (recall that $-C_{p} B_{p} D_{c}$ is a diagonal matrix), we are able to apply Theorem 1 to each component of $e$ and obtain $\bar{\tau}_{1}$ and $\bar{\tau}_{2}$, respectively. Basically, one finds $M_{1}, M_{2}, \lambda_{1}, \lambda_{2}>0$ such that

$$
\begin{aligned}
\left\|e_{1}(t)\right\| & \leq \sqrt{M_{1}}\left\|\psi_{e_{1}}\right\|_{d} e^{-\frac{\lambda_{1}}{2}\left(t-t_{0}\right)} \\
& \leq \sqrt{M_{1}}\left\|\psi_{e}\right\|_{d} e^{-\frac{\lambda_{1}}{2}\left(t-t_{0}\right)},
\end{aligned}
$$




\begin{tabular}{|c|c|c|c|c|}
\cline { 3 - 5 } \multicolumn{2}{c|}{} & \multicolumn{3}{c|}{ UGAS } \\
\cline { 3 - 5 } \multicolumn{2}{c|}{} & $d=0$ & $d=6.3$ & $d=40$ \\
\hline \multirow{3}{*}{ this paper } & no scheduling & $\bar{\tau}=11$ & $\bar{\tau}=8.2$ & $\bar{\tau}=1.4$ \\
\cline { 2 - 5 } & \multirow{2}{*}{ our scheduling } & $\bar{\tau}_{1}=11$ & $\bar{\tau}_{1}=6.1$ & $\bar{\tau}_{1}=1.9$ \\
& & $\bar{\tau}_{2}=11$ & $\bar{\tau}_{2}=7.1$ & $\bar{\tau}_{2}=2.2$ \\
\hline$[7]$ & RR scheduling & $\bar{\tau}_{[7]}=8.9$ & N/A & N/A \\
\hline
\end{tabular}

TABLE I

COMPARISON BETWEen OUR METHOdOLOGY AND [7] FOR UGAS. AlL DELAYS $d$ AND MATIS $\bar{\tau}$ ARE MEASURED IN MILLISECONDS.

\begin{tabular}{|c|c|c|c|}
\cline { 3 - 4 } \multicolumn{2}{c|}{} & \multicolumn{2}{c|}{$\mathcal{L}_{2}$-gain $\gamma_{z}=200$} \\
\cline { 3 - 4 } \multicolumn{2}{c|}{} & $d=10$ & $d=40$ \\
\hline \multirow{3}{*}{ this paper } & no scheduling & $\bar{\tau}=1.7$ & $\bar{\tau}=0.6$ \\
\cline { 3 - 4 } & \multirow{2}{*}{ our scheduling } & $\bar{\tau}_{1}=2$ & $\bar{\tau}_{1}=0.3$ \\
& & $\bar{\tau}_{2}=2$ & $\bar{\tau}_{2}=0.2$ \\
\hline$[7]$ & RR scheduling & N/A & N/A \\
\hline
\end{tabular}

TABLE II

COMPARISON OF OUR METHODOLOGY AND [7] FOR $\mathcal{L}_{2}$-STABILITY. DELAYS $d$ AND MATIS $\bar{\tau}$ ARE EXPRESSED IN MILLISECONDS.

and

$$
\begin{aligned}
\left\|e_{2}(t)\right\| & \leq \sqrt{M_{2}}\left\|\psi_{e_{2}}\right\|_{d} e^{-\frac{\lambda_{2}}{2}\left(t-t_{0}\right)} \\
& \leq \sqrt{M_{2}}\left\|\psi_{e}\right\|_{d} e^{-\frac{\lambda_{2}}{2}\left(t-t_{0}\right)},
\end{aligned}
$$

for all $t \geq t_{0}$, where $\psi_{e_{i}} \in P C\left(\left[t_{0}-d, t_{0}\right], \mathbb{R}\right), i \in\{1,2\}$, is the initial condition of the $i^{\text {th }}$ component of $e$. From the definition of the Euclidean norm, one deduces UGES as follows

$$
\begin{aligned}
\|e(t)\| & \leq \sqrt{M_{1} e^{-\lambda_{1}\left(t-t_{0}\right)}+M_{2} e^{-\lambda_{2}\left(t-t_{0}\right)}}\left\|\psi_{0}\right\|_{d} \\
& \leq \bar{M} e^{-\underline{\lambda}\left(t-t_{0}\right)}\left\|\psi_{0}\right\|_{d},
\end{aligned}
$$

for all $t \geq t_{0}$, where $\bar{M}:=\sqrt{2 \max \left\{M_{1}, M_{2}\right\}}$ and $\underline{\lambda}:=$ $\frac{\min \left\{\lambda_{1}, \lambda_{2}\right\}}{2}$, so that Theorem 2 is applicable. By selecting the intersampling interval equal to $\frac{\min \left\{\bar{\tau}_{1}, \bar{\tau}_{2}\right\}}{2}$, one easily reconstructs the Round-Robin (RR) scheduling, designed on the premise of bus communication, found in [7].

Notice that the maximal possible $\bar{\tau}_{[7]}$ for RR scheduling, obtained with $d=0 \mathrm{~ms}$ and when interested in UGAS, is $8.9 \mathrm{~ms}$. Hence, the maximal theoretical delay that can be considered in [7] for RR scheduling is $d=8.9 \mathrm{~ms}$, i.e., the small delay case. We point out that our methodology is able to consider delays that are significantly greater than $8.9 \mathrm{~ms}$ even for $\mathcal{L}_{p}$-stability (refer to Tables I and II). In fact, provided that $\Sigma_{n}$ is $\mathcal{L}_{p}$-stable for some $d \geq 0$ (each such $d$ is an admissible delay), there exists a stabilizing $\tau>0$ (see Remark 1). As can be concluded from Tables I and II, our MATIs are slightly more conservative than MATIs in [7].

\section{CONCLUSION}

In this paper we propose a framework for computing MATIs of LTV control systems even for the large delay case. Our approach is based on Lyapunov-Razumikhin type of arguments while establishing UGES of impulsive delayed LTV systems. Subsequently, UGES is exploited towards establishing $\mathcal{L}_{p}$-stability with bias of the closed-loop system. The benchmark example of batch reactor illustrates our methodology in detail and suggests that the obtained MATIs for the small-delay case are slightly more conservative in comparison with the related work.

In the future, we plan to integrate scheduling protocols and nonlinear dynamics into our framework. In an effort to extend the obtained MATIs, we plan to consider LyapunovKrasovskii functionals. In addition, communication channels with different and time-varying delays are of interest. Finally, in addition to $\mathcal{L}_{p}$-stability, we plan to address Input-to-State Stability under intermittent transmissions and delays.

\section{REFERENCES}

[1] I. D. Landau and Z. Gianluca, Digital Control Systems: Design, Identification and Implementation, ser. Communications and Control Engineering. London: Springer-Verlag, 2006.

[2] J. Hespanha, P. Naghshtabrizi, and X. Yonggang, "A survey of recent results in Networked Control Systems," Proceedings of the IEEE, vol. 95 , no. 1, pp. 138 - 162, January 2007.

[3] C. Tongwen and B. A. Francis, Optimal sampled-data control systems. London: Springer-Verlag, 1995.

[4] D. Nešić and A. R. Teel, "Input-output stability properties of Networked Control Systems," IEEE Transactions on Automatic Control, vol. 49, no. 10, pp. 1650-1667, October 2004.

[5] C.-Y. Kao and A. Rantzer, "Stability analysis of systems with uncertain time-varying delays," Automatica, vol. 43, no. 6, pp. 959-970, 2007.

[6] S. Hirche, T. Matiakis, and M. Buss, "A distributed controller approach for delay-independent stability of networked control systems," Automatica, vol. 45, no. 5, pp. 1828-1836, August 2009.

[7] W. P. M. H. Heemels, A. R. Teel, N. V. de Wouw, and D. Nešić, "Networked control systems with communication constraints: Tradeoffs between transmission intervals, delays and performance," IEEE Trans. on Automatic Control, vol. 55, no. 8, pp. 1781-1796, 2010.

[8] T. Estrada and P. J. Antsaklis, "Model-based control with intermittent feedback: Bridging the gap between continuous and instantaneous feedback," Intl. Journal of Control, vol. 83, no. 12, pp. 2588-2605, 2010.

[9] R. Postoyan, P. Tabuada, D. Nešić, and A. Anta, "Event-triggered and self-triggered stabilization of distributed networked control systems," in Proceedings of the IEEE Conference on Decision and Control, 2011, pp. $2565-2570$.

[10] D. Tolić and R. Fierro, "Decentralized output synchronization of heterogeneous linear systems with fixed and switching topology via selftriggered communication," in Proceedings of the American Control Conference, 2013, pp. 4655-4660.

[11] H. Yu and P. J. Antsaklis, "Event-triggered output feedback control for networked control systems using passivity: Achieving $\mathcal{L}_{2}$ stability in the presence of communication delays and signal quantization,' Automatica, vol. 49, no. 1, pp. $30-38,2013$.

[12] A. Molin and S. Hirche, "On the optimality of certainty equivalence for event-triggered control systems," IEEE Transactions on Automatic Control, vol. 2, no. 58, pp. 470-474, 2013.

[13] A. Chaillet and A. Bicchi, "Delay compensation in packet-switching networked controlled sytems," in Proceedings of the IEEE Conference on Decision and Control, 2008, pp. 3620-3625.

[14] J. Zhou and Q. Wu, "Exponential stability of impulsive delayed linear differential equations," IEEE Transactions on Circuits and Systems II: Express Briefs, vol. 56, no. 9, pp. 744-748, September 2009.

[15] G. H. Ballinger, "Qualitative theory of impulsive delay differential equations," Ph.D. dissertation, University of Waterloo, Ontario, Canada, 1999.

[16] H. Khalil, Nonlinear Systems, 3rd ed. Prentice Hall, 2002.

[17] Z. P. Jiang, A. R. Teel, and L. Praly, "Small-gain theorem for ISS systems and applications," Mathematics of Control, Signals and Systems, vol. 7, no. 2, pp. 95-120, 1994.

[18] A. Anokhin, L. Berezansky, and E. Braverman, "Exponential stability of linear delay impulsive differential equations," Journal of Mathematical Analysis and Applications, vol. 193, no. 3, pp. 923-941, 1995.

[19] W. Michiels and S. Gumussoy, "Characterization and computation of h-infinity norms of time-delay systems," IEEE Transactions on Automatic Control, vol. 31, no. 4, pp. 2093-2115, 2010. 